\title{
Evaluation and modification of von Kries chromatic adaptation transform
}

\author{
Shining Ma*1, Peter Hanselaer ${ }^{1}$, Kees Teunissen ${ }^{2}$, and Kevin A.G. Smet ${ }^{1}$ \\ ${ }^{1}$ ESAT/Light \& Lighting Laboratory, KU Leuven, Ghent, Belgium \\ ${ }^{2}$ Signify, High Tech Campus 7, Eindhoven, The Netherlands \\ *shining.ma@kuleuven.be
}

\section{Abstract}

Over the years, various chromatic adaptation transforms have been derived to fit the visual perception. However, some research demonstrated that CATO2, the most widely used chromatic adaptation transform, overestimates the degree of adaptation, especially for colored illumination. In this study, a memory color matching experiment was conducted in a real scene with the background adapting field varying in field of view, luminance and chromaticity. It showed that a larger field of view results in more complete adaptation. The results were used to test several existing chromatic adaptation models and to develop three new types of models. All of them improved the performance to some extent, especially for the illuminations with low CCT.

\section{Introduction}

Chromatic adaptation is a perceptual phenomenon that keeps the approximately color appearance constant across changes in illumination. Over the years, many Chromatic Adaptation Transforms (CAT) have been developed. In 1902, von Kries [1] proposed the von Kries coefficient law based on Young-Helmholtz. theory, which states that chromatic adaptation can be considered as a change in the cone sensitivities, whereby each of the cone sensitivities (responses) is independently scaled:

$$
\left(\begin{array}{c}
L_{c} \\
M_{c} \\
S_{c}
\end{array}\right)=\left(\begin{array}{lll}
\alpha & & \\
& \beta & \\
& & \lambda
\end{array}\right)\left(\begin{array}{c}
L \\
M \\
S
\end{array}\right)
$$

$L, M$ and $S$ are the responses of the long, medium and short cones and the subscript $c$ denotes the predicted corresponding color. Because von Kries didn't mention how to calculate these coefficients, CIE adopted a version of the von Kries model whereby the gain control factors are determined using the cone responses of the adopted whites under the two illuminants:

$$
\alpha=\frac{L_{w 2}}{L_{w 1}} \quad \beta=\frac{M_{w 2}}{M_{w 1}} \quad \lambda=\frac{S_{w 2}}{S_{w 1}}
$$

with the subscripts $w 1$ and $w 2$ referring to white points under illuminants 1 and 2.

Equation (1) and (2) are based on the assumption that the adaptation is complete. However, the adaptation process is always incomplete in many viewing conditions. In the CAT02 transform [2], [3], the degree of chromatic adaptation factor $D$, ranging from 0 (no adaptation) to 1 (complete adaptation), is calculated as follows:

$$
D=F\left[1-\left(\frac{1}{3.6}\right) e^{\left(\frac{-L_{a}-42}{92}\right)}\right]
$$

Where $F$ is set to $1.0,0.9$ or 0.8 for 'average', 'dim' or 'dark' surround conditions, respectively and $L_{a}$ is the luminance $\left(\mathrm{cd} / \mathrm{m}^{2}\right)$ of the adapting field. In the CAT02 model, corresponding cone excitations are calculated as in Equation (4):

$$
\left(\begin{array}{c}
L_{c} \\
M_{c} \\
S_{c}
\end{array}\right)=\left[D\left(\begin{array}{lll}
\alpha & & \\
& \beta & \\
& & \lambda
\end{array}\right)+1-D\right]\left(\begin{array}{c}
L \\
M \\
S
\end{array}\right)
$$

Here, $D$ only depends on two factors $\left(F\right.$ and $L_{a}$ ), both related to the luminance dimension of adapting environment. However, several studies show that CAT02 cannot predict corresponding colors accurately, mainly because of an overestimation of the degree of adaption $D$ (Equation (1)). Recently, Smet et al. [4], [5] investigated the impact of the illumination chromaticity on $D$ and demonstrated that the effective $D$ decreases as the illumination becomes more chromatic, but with a clear dependency on chromatic direction. It was also found that the effective degree of adaptation was much less than expected, even under a high luminance neutral background; probably due to the dark surround effectively reducing the adapting luminance of the $50^{\circ}$ background.

In contrast to the von Kries type linear rescaling of the cone fundamental responses, several CATs have the $\mathrm{S}$ cone response undergoing a nonlinear compression (exponent $p$ ). Bartleson [6], [7] derived a transformation to fit the visual data collected using a magnitude estimation technique. The $\mathrm{S}$ cone response was modified through a power transform, while $\mathrm{L}$ and $\mathrm{M}$ cone responses still followed the von Kries coefficient law. Lam and Rigg [8] derived their BFD transform based on the data from a memory matching experiment. The structure of the BFD transform is similar to that of Bartleson's. It was later enhanced by Luo and Hunt [9] to become the CMCCAT97:

$$
\begin{aligned}
& \left(\begin{array}{c}
L_{c} \\
M_{c} \\
S_{c}
\end{array}\right)=\left[D\left(\begin{array}{lll}
\alpha & & \\
& \beta & \\
& & \lambda^{\prime \prime}
\end{array}\right)+1-D\right]\left(\begin{array}{c}
L \\
M \\
S^{p}
\end{array}\right) \\
& p=\left(\frac{S_{r w}}{S_{w}{ }^{p}}\right)^{0.0834}=\left(\frac{1}{\lambda}\right)^{0.0834}
\end{aligned}
$$

Both the Bartleson and CMCCAT97 models treated S cone specially, because its mechanism of color vision consistently seems to behave differently from the $\mathrm{L}$ cone and $\mathrm{M}$ cone mechanism [10][13].

To further study other factors that may influence chromatic adaptation, several achromatic matching experiments were conducted for 13 different illumination chromaticities and with the background field of view varying between $20^{\circ}$ and $80^{\circ}$. In one set of experiments, the background luminance was kept fixed at 180 $c d / m^{2}$, while in another its luminance was changed as to generate a constant corneal illuminance of $7 l x$. Based on these visual data, three modified models were proposed to improve CAT 
performance. Furthermore, the three models were evaluated and compared.

\section{Experiment Design}

In the experiments, observers viewed a real 3D scene composed of a spectrally neutral (non-fluorescent) grey cube and a white background, providing the adaptation field. The area outside of the background was dark. The grey cube had a $6^{\circ}$ field of view and was centrally positioned in the background scene. A calibrated data projector provided independently controllable illumination for the background and object. The reflectance spectra of the background and the object, as measured by a Hunterlab UltraScan Pro colorimeter, are plotted in Figure 1. The spectral radiance of the background and cube was measured with a calibrated OceanOptics QE65Pro tele-spectroradiometer. All colorimetric calculations were done using the CIE $196410^{\circ}$ color matching functions as the stimulus size is larger than $4^{\circ}$.

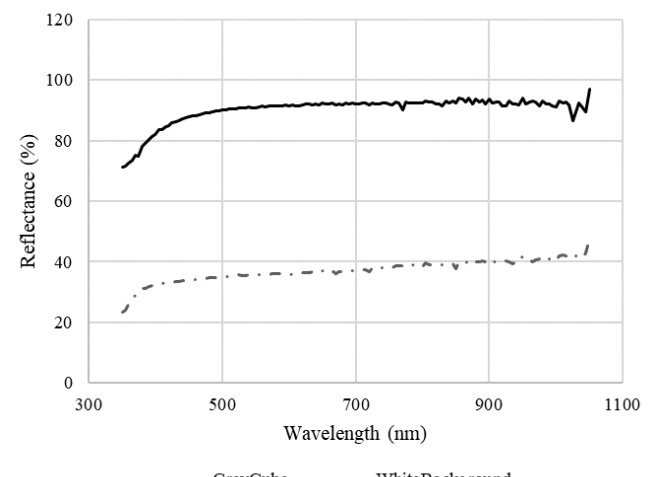

Figure 1. Reflectance spectra of the grey cube stimulus (dash line) and the background (solid line).

(a)

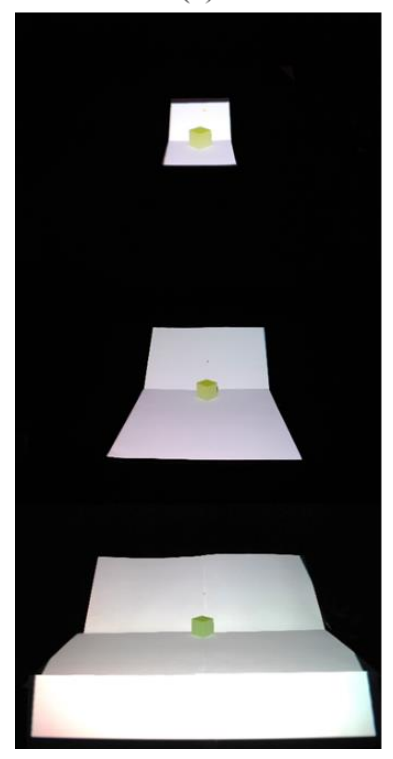

Figure 2. The adapting fields in the real scene. (a) Equal luminance backgrounds. Top, middle and bottom correspond to $20^{\circ}, 40^{\circ}, 80^{\circ}$ respectively. (b) Equal vertical illuminance at eye position (corneal illuminance). Top, middle and bottom correspond to $20^{\circ}, 40^{\circ}, 60^{\circ}$ respectively.
As shown in Figure 2, the experiment was conducted under two groups of adapting fields. In both groups the field of view was varied, but in one the background luminance was kept fixed at 180 $\mathrm{cd} / \mathrm{m}^{2}$, while in the other the vertical illuminance level at eye level (corneal illuminance) was kept constant at $7 l x$. The field sizes of the two groups were $20^{\circ}, 40^{\circ}, 80^{\circ}$ and $20^{\circ}, 40^{\circ}, 60^{\circ}$, respectively. At $7 l x$ corneal illuminance, the latter field sizes resulted in background luminance values of $180 \mathrm{~cd} / \mathrm{m}^{2}, 35 \mathrm{~cd} / \mathrm{m}^{2}$ and 20 $c d / m^{2}$, respectively. Each combination of size and luminance level of the adapting field was an experiment session. In total, there were five sessions as the $20^{\circ}$ field size at $180 \mathrm{~cd} / \mathrm{m}^{2}$ was common to both groups. For each field size and luminance level, achromatic matches were collected for thirteen different chromaticities of the adapting field: three neutral ones and ten more colored chromaticities. The neutral ones had chromaticities corresponding to CIE illuminants E and D65, and the most neutral white $(\mathrm{N})$ found by Smet et al. [14], [15]. The colored chromaticties corresponded to those of five Planckian radiators of CIE illuminant A, 2000K (P2k), 4000K (P4k), 12000K (P12k) and infinite K (Pinf), and five high chromatic sources (Red, Yellow, Green, Blue, and Purple). As can be seen from Figure 3, the distribution of the thirteen background chromaticities, plotted in CIE 1976 u $_{10}$ 'v $_{10}$ ' color space, covers a wide color gamut.

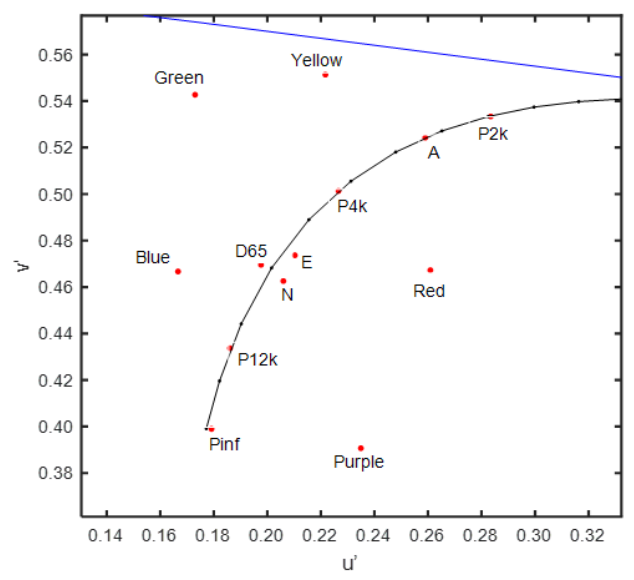

Figure 3. The distribution of thirteen background chromaticities in the CIE 1976 u' $_{10}$ '10 diagram.

During the experiment, observers were instructed to adjust the color of the 3D cube until it appears neutral grey (achromatic setting), by navigating in the CIE 1976 u'$_{10} v^{\prime}{ }_{10}$ space using the 4 arrow keys (up, down, left, right) of a regular keyboard. Note that the luminance of the stimulus was kept constant during the achromatic matching. For each background/illumination condition, to minimize the starting point bias, four matches were made starting from different, highly saturated stimulus chromaticities that were symmetrically distributed along the hue circle centered at the chromaticity of the equal-energy white. Chromaticities of each match were calculated from their spectral radiance measured by an OceanOptics QE65Pro tele-spectroradiometer. To ensure a near steady-state adaptation state of each observer, they were asked to adapt to the current illumination for $45 \mathrm{~s}$ before starting their match [16]. Illumination chromaticities and starting points were presented randomly within each session. To avoid order bias, the five sessions were randomly varied among observers. 
Eleven observers (7 females, 4 males) with normal vision, as tested by the Ishihara 24-plate test, participated the experiment. In total, 2860 matching were made ( 1 target object $\times 11$ observers $\times 13$ background chromaticities $\times 4$ starting points $\times 5$ sessions).

\section{Results}

\section{Observer variability}

The inter-observer variability and intra-observer variability were evaluated in terms of the mean color difference with the mean $(M C D M)$ [17]. Higher $M C D M$ value corresponds to larger observer variation (inter-variability), and lower repeatability (intravariability). The inter-observer variability $M C D M$ value was calculated as the color difference in CIE $1976 u_{10} v_{10}$ ' color space between the individual observer's average match from 4 starting points and the average match of all the observers. The intravariability $M C D M$ for each background condition has been evaluated using the color differences between the matches resulting from each of the four starting points and their average.

For the equal luminance group, the intra- and inter-observer variability in terms of the $M C D M$ values for the $20^{\circ}, 40^{\circ}$ and $80^{\circ}$ adapting fields are $(0.0097,0.0094,0.0089)$ and $(0.0095,0.0096$, $0.0066)$, respectively. For the equal illuminance group, the intraand inter-observer variability for the $20^{\circ}, 40^{\circ}$ and $60^{\circ}$ adapting field are $(0.0097,0.0075,0.0094)$ and $(0.0095,0.0100,0.0081)$, respectively.

\section{Model performance test}

For each background luminance/field size condition, 12 pairs of corresponding colors were generated from the 13 background chromaticities by selecting the chromaticity of $\mathrm{N}$ as the neutral reference. A total of 60 (12 chromaticities $\times 5$ conditions) corresponding color pairs were obtained. These were used to calculate an effective (optimized) degree of chromatic adaptation $D$ using Equation (4) by minimizing the $D E u^{\prime} 10 v^{\prime} 10$ between the predicted corresponding color and the visual match made under the reference background chromaticity $(\mathrm{N})$. The Hunt-Pointer-Estevez, (HPE) transformation matrix [18] from XYZ to LMS cone space was used as sensor space, instead of the empirically determined CAT02 sensors.

Table 1 shows the optimized $D$ values of the 13 background chromaticities for the equal luminance and equal illuminance groups. Note that the $D$ value for background chromaticity $\mathrm{N}$ is equal to one as it was set as the reference white (baseline) for the chromatic adaptation transform. From the table, several trends can be observed for the optimized $D$. Firstly, comparing different chromaticities, higher chroma of the background results in lower optimized $D$, which indicates more incomplete adaptation state. Secondly, the impact is more pronounced along the yellowish direction than the bluish direction, which is consistent with the results from Smet et al [4], [5]. Thirdly, for the equal luminance group, larger fields of view, generating a more immersive environment, generally result in a more complete adaptation state. Exceptions, such as the lower $D$ for $80^{\circ}$ compared to the $D$ for $40^{\circ}$, observed under neutral chromaticities (E, D65) are likely the result from their closeness to the reference chromaticity $(\mathrm{N})$, which causes the optimized $D$ to be easily influenced by observer variability. Fourthly, for the equal illuminance group, similar trends can be observed. Lower optimized $D$ for more chromatic backgrounds, but also, surprisingly, increasing $D$ for increasing field size and this despite the dramatic drops in adapting luminance (e.g. $20^{\circ}$ versus $60^{\circ}$ corresponds to a reduction by a factor or more than 4). Fifthly, differences between $D$ values for $20^{\circ}$ and $40^{\circ}$ are more pronounced than those between $40^{\circ}$ and $60^{\circ}$, suggesting a diminishing effect of background size on the degree of adaptation for large enough backgrounds. It is as yet unclear what could be the underlying cause.

Table 1. Summary of the optimized $D$ under the 13 background chromaticities for the equal luminance and equal illuminance background conditions. The first group includes 3 levels for the field of view with adapting luminance at $180 \mathrm{~cd} / \mathrm{m}^{2}$. The second group also includes 3 levels of field of view: $20^{\circ}\left(180 \mathrm{~cd} / \mathrm{m}^{2}\right)$, $40^{\circ}\left(35 \mathrm{~cd} / \mathrm{m}^{2}\right)$ and $60^{\circ}\left(20 \mathrm{~cd} / \mathrm{m}^{2}\right)$

\begin{tabular}{|c|ccc|ccc|}
\hline \multirow{2}{*}{$\begin{array}{c}\text { Background } \\
\text { chrom. }\end{array}$} & \multicolumn{3}{|c|}{$\begin{array}{c}\text { Equal luminance } \\
\text { group }\end{array}$} & \multicolumn{3}{c|}{$\begin{array}{c}\text { Equal illuminance } \\
\text { group }\end{array}$} \\
\cline { 2 - 7 } & $20^{\circ}$ & $40^{\circ}$ & $80^{\circ}$ & $20^{\circ}$ & $40^{\circ}$ & $60^{\circ}$ \\
\hline $\mathrm{N}$ & 1.00 & 1.00 & 1.00 & 1.00 & 1.00 & 1.00 \\
$\mathrm{E}$ & 0.56 & 0.80 & 0.62 & 0.56 & 0.56 & 0.64 \\
D65 & 0.62 & 0.77 & 0.67 & 0.62 & 0.64 & 0.72 \\
$\mathrm{~A}$ & 0.29 & 0.43 & 0.51 & 0.29 & 0.36 & 0.34 \\
B2K & 0.23 & 0.30 & 0.45 & 0.23 & 0.30 & 0.28 \\
B4K & 0.41 & 0.61 & 0.66 & 0.41 & 0.56 & 0.56 \\
B12K & 0.60 & 0.68 & 0.74 & 0.60 & 0.64 & 0.68 \\
Binf & 0.54 & 0.66 & 0.77 & 0.54 & 0.68 & 0.68 \\
Yellow & 0.09 & 0.16 & 0.21 & 0.09 & 0.13 & 0.18 \\
Green & 0.22 & 0.27 & 0.36 & 0.22 & 0.24 & 0.26 \\
Blue & 0.55 & 0.65 & 0.76 & 0.55 & 0.61 & 0.62 \\
Purple & 0.48 & 0.62 & 0.71 & 0.48 & 0.59 & 0.60 \\
Red & 0.34 & 0.52 & 0.60 & 0.34 & 0.43 & 0.48 \\
Average & 0.46 & 0.57 & 0.62 & 0.46 & 0.52 & 0.54 \\
\hline
\end{tabular}

Table 2 shows the minimized $D E u^{\prime} 10 v^{\prime} 10$ of the 13 backgrounds for the equal luminance and equal illuminance groups. The $D E$ ' $^{\prime} 10 v^{\prime} 10$ value for background chromaticity $\mathrm{N}$ is equal to zero as it was set as the reference white. Firstly, from Table 2, it can be observed that for both equal luminance and equal illuminance groups, yellowish backgrounds located on the Planckian locus (A and $\mathrm{B} 2 \mathrm{~K}$ ) has substantially higher prediction error than other backgrounds. Additionally, the DEu'1ov'10 of neutral (E, D65, B4K) and bluish backgrounds (B12K, Binf, Blue) is smaller than others, sometimes even negligible. In other words, von Kries model with optimized $D$ has good performance for the neutral and bluish backgrounds, but fairly poor performance for the high chroma backgrounds along the yellowish direction. Secondly, there is no substantial difference between the $D E u^{\prime} 10 v^{\prime} 10$ of different field of view. Even though for each backgrounds, the $D E u^{\prime} 10 v^{\prime} 10$ values of different field of view are not exactly the same, their average $D E u^{\prime}{ }_{10} v^{\prime}{ }_{10}$ of all the illuminants are very close, varying from 0.0037 to 0.0044 . 
Table 2. Summary of the minimized $D E u^{\prime}{ }_{10} V^{\prime}{ }_{10}$ under the 13 background chromaticities for the equal luminance and equal illuminance background conditions. The first group includes 3 levels for the field of view with adapting luminance at $180 \mathrm{~cd} / \mathrm{m}^{2}$. The second group also includes 3 levels of field of view: $20^{\circ}(180$ $\left.\mathrm{cd} / \mathrm{m}^{2}\right), 40^{\circ}\left(35 \mathrm{~cd} / \mathrm{m}^{2}\right)$ and $60^{\circ}\left(20 \mathrm{~cd} / \mathrm{m}^{2}\right)$.

\begin{tabular}{|c|ccc|ccc|}
\hline \multirow{2}{*}{$\begin{array}{c}\text { Background } \\
\text { chrom. }\end{array}$} & \multicolumn{3}{|c|}{$\begin{array}{c}\text { Equal luminance } \\
\text { group } \\
\left(\times 10^{-3}\right)\end{array}$} & \multicolumn{3}{c|}{$\begin{array}{c}\text { Equal illuminance } \\
\text { group } \\
\left(\times 10^{-3}\right)\end{array}$} \\
\cline { 2 - 7 } & $20^{\circ}$ & $40^{\circ}$ & $80^{\circ}$ & $20^{\circ}$ & $40^{\circ}$ & $60^{\circ}$ \\
\hline $\mathrm{N}$ & 0.0 & 0.0 & 0.0 & 0.0 & 0.0 & 0.0 \\
EEW & 0.6 & 0.3 & 0.1 & 0.6 & 0.7 & 0.7 \\
D65 & 4.0 & 0.4 & 0.1 & 4.0 & 2.6 & 0.3 \\
$\mathrm{~A}$ & 7.9 & 8.4 & 10.6 & 7.9 & 9.1 & 9.2 \\
B2K & 17.1 & 17.4 & 18.7 & 17.1 & 16.6 & 16.7 \\
B4K & 0.8 & 0.6 & 1.9 & 0.8 & 2.4 & 0.5 \\
B12K & 0.4 & 1.3 & 1.3 & 0.4 & 0.5 & 0.8 \\
Binf & 2.4 & 2.5 & 1.5 & 2.4 & 4.0 & 3.0 \\
Yellow & 2.5 & 5.7 & 7.7 & 2.5 & 8.0 & 4.5 \\
Green & 4.8 & 7.3 & 6.0 & 4.8 & 6.9 & 5.4 \\
Blue & 1.7 & 0.5 & 1.0 & 1.7 & 0.3 & 0.7 \\
Purple & 5.6 & 6.6 & 1.0 & 5.6 & 5.3 & 5.1 \\
Red & 0.7 & 4.2 & 2.3 & 0.7 & 1.2 & 2.3 \\
Average & 3.7 & 4.2 & 4.0 & 3.7 & 4.4 & 3.8 \\
\hline
\end{tabular}

\section{Model development}

Both CMCCAT97 and Bartleson model adopted an extra factor $p$ in the gain control of $\mathrm{S}$ cone excitation. Inspired by that, a compression factor $p$ was added in the $\mathrm{S}$ cone rescaling formula. Three types of modifications are proposed and evaluated.

The first model (M1) adds a power function to the $\mathrm{S}$ cone response, while keeping the gain control coefficients (Equation (2)), as shown below:

$$
\begin{aligned}
& \left(\begin{array}{c}
L_{c} \\
M_{c} \\
S_{c}
\end{array}\right)=\left[D\left(\begin{array}{lll}
\alpha & & \\
& \beta & \\
& & \lambda
\end{array}\right)+1-D\right]\left(\begin{array}{c}
L \\
M \\
S^{p}
\end{array}\right) \\
& p=\left(\frac{S_{w}}{S_{r w}}\right)^{q}=\left(\frac{1}{\lambda}\right)^{q}
\end{aligned}
$$

Where $p$ is the $\mathrm{S}$ cone component and $q$ is a constant to be optimized and other factors have been explained in Equation (1), (2) and (4). Note that the $D$ factor is not calculated by the $C A T O 2 D$ formula, but is optimized as explained below.

The structure of the second model (M2) is identical to the CMCCAT97, it includes a modified gain control coefficient for the S-cones and a S-cone exponent $p$ :
$\left(\begin{array}{c}L_{c} \\ M_{c} \\ S_{c}\end{array}\right)=\left[\left(\begin{array}{lll}\alpha & & \\ & \beta & \\ & & \lambda^{\prime \prime}\end{array}\right)+1-D\right]\left(\begin{array}{c}L \\ M \\ S^{p}\end{array}\right)$

$\lambda "=\frac{S_{r w}}{S_{w}{ }^{p}}$

$p=\left(\frac{S_{w}}{S_{r w}}\right)^{q}=\left(\frac{1}{\lambda}\right)^{q}$

The third model (M3) is still a strong von Kries model with an independent gain control of all three cones. However, the gain control of $\mathrm{S}$ cone is nonlinearly transformed using a power function:

$$
\begin{aligned}
& \left(\begin{array}{c}
L_{c} \\
M_{c}
\end{array}\right)=\left[D\left(\begin{array}{ll}
\alpha & \\
& \beta
\end{array}\right)+1-D\right]\left(\begin{array}{c}
L \\
M
\end{array}\right) \\
& S_{c}=(D \lambda+1-D)^{p} S \\
& p=\left(\frac{S_{w}}{S_{r w}}\right)^{q}=\left(\frac{1}{\lambda}\right)^{q}
\end{aligned}
$$

Table 3. The performance $\left(D E u^{\prime}{ }_{10 V}{ }^{\prime} 10\right)$ of the optimized $D$ for the typically used model (Equation (4)) and three modified models M1, M2, M3. The DEu' $10 V^{\prime}{ }_{10}$ for each background chromaticity is the RMS over the 5 adapting fields with different luminance or size levels. The last row is the RMS of all the 13 background chromaticities in each column. The lowest values are indicated in bold. The highest values are underlined.

\begin{tabular}{|c|c|c|c|c|}
\hline Illuminant & Original & $\mathrm{M} 1$ & $\mathrm{M} 2$ & $\mathrm{M} 3$ \\
\hline $\mathrm{N}$ & $\mathbf{0 . 0 0 0 0}$ & $\mathbf{0 . 0 0 0 0}$ & $\mathbf{0 . 0 0 0 0}$ & $\mathbf{0 . 0 0 0 0}$ \\
$\mathrm{E}$ & $\mathbf{0 . 0 0 0 5}$ & $\underline{0.0013}$ & 0.0006 & 0.0006 \\
D65 & $\underline{0.0021}$ & $\mathbf{0 . 0 0 1 8}$ & 0.002 & $\underline{0.0021}$ \\
$\mathrm{~A}$ & $\underline{0.0091}$ & 0.0032 & 0.0019 & $\mathbf{0 . 0 0 1 4}$ \\
B2K & $\underline{0.0173}$ & 0.0092 & $\mathbf{0 . 0 0 2 0}$ & 0.0039 \\
B4K & 0.0015 & $\underline{0.0024}$ & $\mathbf{0 . 0 0 0 8}$ & 0.0012 \\
B12K & 0.0009 & $\underline{0.0040}$ & 0.0009 & $\mathbf{0 . 0 0 0 6}$ \\
Binf & 0.0028 & $\underline{0.0085}$ & 0.0024 & $\mathbf{0 . 0 0 1 2}$ \\
Yellow & $\underline{0.0060}$ & $\underline{0.0044}$ & 0.0023 & $\mathbf{0 . 0 0 2 2}$ \\
Green & $\underline{0.0061}$ & 0.0042 & 0.0059 & $\mathbf{0 . 0 0 3 4}$ \\
Blue & 0.0010 & $\underline{0.0012}$ & $\mathbf{0 . 0 0 0 8}$ & 0.0010 \\
Purple & 0.0051 & 0.0079 & $\underline{0.0109}$ & $\mathbf{0 . 0 0 3 9}$ \\
Red & $\mathbf{0 . 0 0 2 5}$ & $\underline{0.0041}$ & 0.0033 & 0.0027 \\
RMS & $\underline{0.0062}$ & 0.0049 & 0.0038 & $\mathbf{0 . 0 0 2 2}$ \\
\hline
\end{tabular}

For M1, M2 and M3, $D$ was optimized for each background chromaticity and adapting field extent, while $q$ was a constant optimized over all conditions. The optimized $q$ for M1, M2 and M3 
are $0.0393,0.6116$ and 0.2467 respectively. The performance of the three modified models and the original model shown in Equation (4) are summarized in Table 3. The minimized DEu' $10 v^{\prime} 10$ listed in the table are the RMS (Root Mean Square) over all the 5 adapting field extents under each of the thirteen background chromaticities. It shows that all the three models substantially improve the performance of yellowish illuminants (A, B2K, Yellow). However, for the backgrounds with large $\mathrm{S}$ cone excitation including $\mathrm{B} 12 \mathrm{~K}$, Binf, Purple and Blue, M3 has slightly better performance while M1 and $\mathrm{M} 2$ have similar or worse performance compared to the original one. Of the three models, M2 and M3 perform better than M1, especially for illuminants $\mathrm{B} 2 \mathrm{~K}, \mathrm{~B} 4 \mathrm{~K}, \mathrm{~B} 12 \mathrm{~K}$, and Binf which are located on the Planckian locus. M3 has overall a better performance than M2, especially for illuminants Binf, Green and Purple. Therefore, M3 performs best comparing to M1 and M2. Furthermore, because M3 only applied the compression factor $p$ in the gain control coefficients of $\mathrm{S}$ cone, it still follows the von Kries coefficient law, and can therefore be more easily adopted in the current CAT model.

\section{Conclusion}

An achromatic matching experiment was conducted to collect corresponding color under 13 background chromaticities and 5 adapting fields with different luminance or field sizes. Five adapting fields were divided into two groups: 1) equal luminance $\left(180 \mathrm{~cd} / \mathrm{m}^{2}\right)$ at $\left.20^{\circ}, 40^{\circ}, 80^{\circ} ; 2\right)$ equal vertical illuminance at $20^{\circ}\left(180 \mathrm{~cd} / \mathrm{m}^{2}\right)$, $40^{\circ}\left(35 \mathrm{~cd} / \mathrm{m}^{2}\right), 60^{\circ}\left(20 \mathrm{~cd} / \mathrm{m}^{2}\right)$.

Firstly, the impact of adapting field size has been investigated. Results from both groups indicate that larger fields lead to higher optimized $D$ values, corresponding to a more complete adaptation state. In the second group, even though the luminance of the $60^{\circ}$ adapting field was 8 times lower than $20^{\circ}$ adapting field, larger background size still had higher $D$ values.

Secondly, the von Kries model with optimized $D$ has good performance for neutral and bluish backgrounds with small $D E u^{\prime} 10 v^{\prime} 10$, but fairly poor performance for the yellowish backgrounds along the Planckian locus.

Thirdly, three models were developed based on the typical von Kries model, presented in Equation (4). All the three models outperformed the original model under most illuminants, especially the yellowish illuminants, but not under bluish illuminants. Overall, M3 performs best comparing to M1 and M2. In addition, M3 is more easily adopted than $\mathrm{M} 2$ because the $\mathrm{S}$ cone remains linearly gain controlled in M3 in comparison with the $\mathrm{L}$ and $\mathrm{M}$ cone responses. These results can provide insights for the further development of more accurate and more comprehensive CAT models.

\section{References}

[1] Commission Internationale de l'Eclairage, “CIE 160:2004 A Review of Chromatic Adaptation Transforms," 2004.

[2] M. R. Luo, "A review of chromatic adaptation," Rev. Prog. Color. Relat. Top., vol. 30(1), pp. 77-92, 2000.

[3] N. Moroney, M. D. Fairchild, R. W. G. Hunt, C. Li, M. R. Luo, and T. Newman, "The CIECAM02 Color Appearance Model," IS \&T/SID Tenth Color Imaging Conf., pp. 23-27, 2002.

[4] K. A. G. Smet, Q. Zhai, M. R. Luo, and P. Hanselaer, "Study of chromatic adaptation using memory color matches, Part I: neutral illuminants," Opt. Express, 2017.

[5] K. A. G. Smet, Q. Zhai, M. R. Luo, and P. Hanselaer, "Study of chromatic adaptation using memory color matches, Part II: colored illuminants," Opt. Express, 2017.

[6] C. J. Bartleson, "A review of chromatic adaptation," AIC Proceedings, Color, vol. 77, pp. 63-96, 1978.

[7] C. J. Bartleson, "Changes in color appearance with variations in chromatic adaptation," Color Res. Appl., vol. 4, no. 3, pp. 119-138, 1979.

[8] K. M. Lam, Metamerism and colour constancy. Ph.D. thesis, vol. Ph.D. 1985.

[9] M. R. Luo and R. W. G. Hunt, "A chromatic adaptation transform and a colour inconstancy index," Color Res. Appl., vol. 23, no. 3, pp. 154-158, 1998.

[10] J. Baudin, J. M. Angueyra, R. Sinha, and F. Rieke, "S-cone photoreceptors in the primate retina are functionally distinct from $\mathrm{L}$ and M cones," Elife, vol. 8, pp. 1-22, 2019.

[11] A. Stockman, M. Langendörfer, and L. T. Sharpe, "Human shortwavelength-sensitive cone light adaptation," J. Vis., vol. 7, no. 3, p. 4, 2007.

[12] A. Stockman, D. I. A. MacLeod, and D. D. DePriest, "The temporal properties of the human short-wave photoreceptors and their associated pathways," Vision Res., vol. 31, no. 2, pp. 189-208, 1991.

[13] H. E. Smithson and J. D. Mollon, "Is the S-opponent chromatic subsystem sluggish?," Vision Res., vol. 44, no. 25, pp. 2919-2929, 2004.

[14] K. A. G. Smet, G. Deconinck, and P. Hanselaer, "Chromaticity of unique white in illumination mode," vol. 23 , no. 10, pp. 12488 12495, 2015.

[15] K. A. G. Smet, "Two Neutral White Illumination Loci Based on Unique White Rating and Degree of Chromatic Adaptation," LEUKOS - J. Illum. Eng. Soc. North Am., vol. 14, no. 2, pp. 55-67, 2018.

[16] M. D. Fairchild and L. Reniff, "Time-course of chromatic adaptation for color-appearance judgments," J. Opt. Soc. Am. a-Optics Image Sci. Vis., vol. 12, no. 5, pp. 824-833, 1995.

[17] F. W. Billmeyer and P. J. Alessi, "Assessment of Color-Measuring Instruments,” Color Res. Appl., vol. 6, no. 4, pp. 195-202, 1981.

[18] M. D. Fairchild, Color Appearance Models, 2nd ed. Chichester: John Wiley \& Sons, 2005.

\section{Author Biography}

Shining Ma received her BS and MS in optical engineering from Zhejiang University (2017). Now she is a PhD researcher in ESAT/Light \& Lighting Laboratory, KU Leuven. Her PhD subject is 'Chromatic adaptation in the complex scene. 